\title{
Long-Range Magnetic Interactions in the Multiferroic Antiferromagnet $\mathrm{MnWO}_{4}$
}

\author{
Feng Ye,, , * Randy S. Fishman, ${ }^{2}$ Jaime A. Fernandez-Baca, ${ }^{1,3}$ Andrey A. Podlesnyak, ${ }^{1}$ \\ Georg Ehlers, ${ }^{1}$ Herbert A. Mook, ${ }^{1}$ Yaqi Wang, ${ }^{4}$ Bernd Lorenz,${ }^{4}$ and C. W. Chu ${ }^{4}$ \\ ${ }^{1}$ Neutron Scattering Science Division, Oak Ridge National Laboratory, Oak Ridge, Tennessee 37831, USA \\ ${ }^{2}$ Materials Science and Technology Division, Oak Ridge National Laboratory, Oak Ridge, Tennessee 37831, USA \\ ${ }^{3}$ Department of Physics and Astronomy, The University of Tennessee, Knoxville, Tennessee 37996, USA \\ ${ }^{4}$ Department of Physics and TCSUH, University of Houston, Houston, Texas 77204, USA
}

(Dated: April 25, 2022)

\begin{abstract}
The spin-wave excitations of the multiferroic $\mathrm{MnWO}_{4}$ have been measured in its low-temperature collinear commensurate phase using high-resolution inelastic neutron scattering. These excitations can be well described by a Heisenberg model with competing long-range exchange interactions and a single-ion anisotropy term. The magnetic interactions are strongly frustrated within the zigzag spin chain along $c$ axis and between chains along the $a$ axis, while the coupling between spin along the $b$ axis is much weaker. The balance of these interactions results in the noncollinear incommensurate spin structure associated with the magnetoelectric effect, and the perturbation of the magnetic interactions leads to the observed rich phase diagrams of the chemically-doped materials. This delicate balance can also be tuned by the application of external electric or magnetic fields to achieve magnetoelectric control of this type of materials.

PACS numbers: 78.70.Nx, 75.30.Ds, 75.85.+t
\end{abstract}

Magnetoelectric multiferroic materials, which exhibit the coexistence of ferroelectric (FE) and magnetic orders, have attracted great attention in recent years $\underline{1}^{-\underline{4}}$ Several classes of multiferroics among transition metal oxides have been discovered including geometrically-frustrated

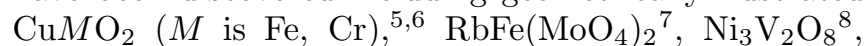
or rare-earth $(R)$ manganites $R \mathrm{MnO}_{3}$ and $R \mathrm{Mn}_{2} \mathrm{O}_{5} \cdot \underline{9^{-11}}$ The ability to simultaneously control the electric (E) and magnetic (M) properties makes these multiferroics promising candidates for technological applications $\frac{9.12}{.12}$ A characteristic feature in those magnetically induced multiferroics is the presence of long-range magnetic structures with noncollinear spiral spin configurations. Such magnetic order is a consequence of magnetic frustration either due to geometric constraints or competing exchange interactions resulting in a close competition of different magnetic structures that are nearly degenerate in energy.

The mineral Hübnerite $\mathrm{MnWO}_{4}$ appears to be an unique material that not only exhibits intriguing multiferroic phenomena but also shows rich magnetic phases via chemical substitutions $\underline{\underline{13}-21}$ It has been considered one of the prototypical multiferroics capable of magnetoelectric (ME) control. 16,22 Unlike $R \mathrm{MnO}_{3}$ where the spiral magnetic structure often involves ordering of the rare-earth moments, $\mathrm{MnWO}_{4}$ is a frustrated antiferromagnet (AF) with only one type of magnetic ion. The $\mathrm{Mn}^{2+}$ spins $(S=5 / 2)$ undergo successive transitions in zero field ${ }^{23}$ The low-temperature $(T)$ magnetic structure has a collinear spin configuration [Fig. 1(a)]. For $T$ between $7.8 \mathrm{~K}\left(T_{N 1}\right)$ and $12 \mathrm{~K}\left(T_{N 2}\right)$, the magnetic structure evolves into an incommensurate (ICM) elliptical spiral configuration accompanied by a spontaneous electric polarization $P$ along the crystalline $b$ axis. When $T$ is further raised between $T_{N 2}$ and $T_{N 3}(\approx 13.5 \mathrm{~K}), \mathrm{MnWO}_{4}$ becomes a collinear ICM and paraelectric.
In $\mathrm{MnWO}_{4}$, the electric polarization that is correlated with the spiral magnetic structure can be well understood by the microscopic picture regarded as inverse Dzyaloshinskii-Moriya interaction. ${ }^{24-26}$ On the other hand, characterizing the magnetic interactions that cause the formation of the complex spin structures and understanding how the modification of exchange couplings affects the evolution between different phases remains an unresolved issue despite intensive experimental and theoretical studies $\underset{27-29}{ }$ Early inelastic neutron scattering (INS) work suggested that the stabilization of the collinear configuration requires higher order magnetic interactions. $\stackrel{27}{ }$ Although the magnetic exchange parameters obtained by Ehrenberg et al. fit the experimental data, the longest bond distance was associated with the strongest coupling constant. Later, density functional calculation and classical spin analysis were performed to investigate the magnetic structure and $\mathrm{FE}$ polarization in $\mathrm{MnWO}_{4}, \stackrel{28}{=}$ Tian et al. concluded that the spin exchange interactions are frustrated along both the $a$ and $c$ axes. However, a quantitative experimental characterization of the magnetic interactions is still lacking. Here we report high-resolution INS measurements that show that the low- $T$ magnetic ground state of pure $\mathrm{MnWO}_{4}$ indeed results from the competition of long-range interactions that are highly frustrated and sensitive to small perturbations. The comprehensive mapping of the of the magnetic excitations along several symmetry directions allowed an unambiguous determination of the dispersion relations and the exchange interactions. Most importantly, such microscopic characterization of the spin coupling constants provides a fundamental step toward the construction of the ground state Hamiltonian from which the FE phase can be derived.

A 5-g single crystal of $\mathrm{MnWO}_{4}$ was grown by the floating-zone technique. Neutron diffraction was per- 

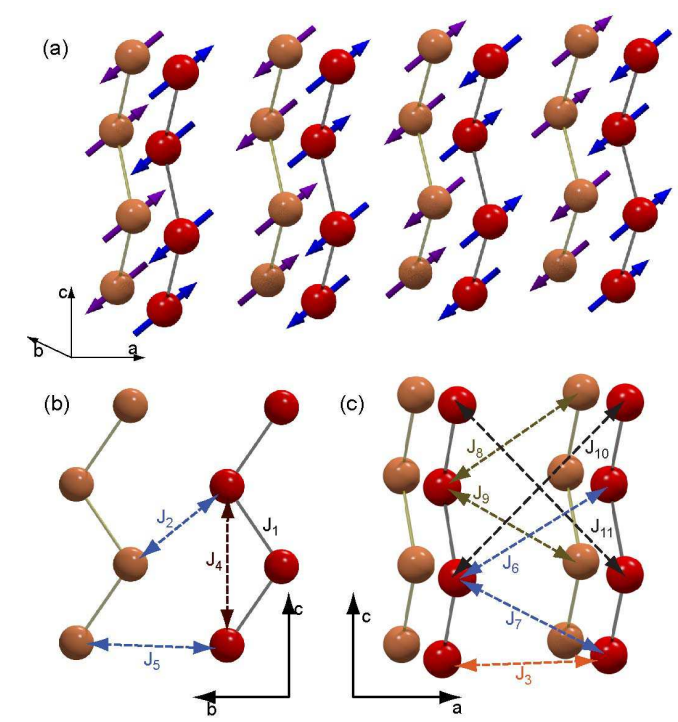

FIG. 1: (Color online) (a) The magnetic structure of $\mathrm{MnWO}_{4}$ in the collinear, commensurate phase at low temperature. The magnetic spins lie in the $a c$ plane with the moment canted to the $a$ axis about $35^{\circ} .^{23}$ The magnetic spins form zigzag $\uparrow \uparrow \downarrow \downarrow$ chains along the $c$ axis, and are coupled antiferromagnetically along the $b$ axis. (b) The magnetic interactions along and between spin chains in the $b c$ plane. (c) Higher order magnetic interactions along the $a$ axis direction. The magnetic couplings are labeled with increasing bonding distance. Note the monoclinic crystal structure $\left(\beta=91.14^{\circ}\right)$ makes $J_{6} / J_{7}, J_{8} / J_{9}, J_{10} / J_{11}$ pairs different.

formed on a small piece of this crystal $(0.2 \mathrm{~g})$ to verify the spin structure using the four-circle single crystal diffractometer HB3A at the High Flux Isotope Reactor at the Oak Ridge National Laboratory (ORNL). The inelastic neutron scattering measurements were performed using the Cold Neutron Chopper Spectrometer (CNCS) at the Spallation Neutron Source at ORNL. The momentum transfer wavevectors $q=\left(q_{x}, q_{y}, q_{z}\right)$ are in units of $\AA^{-1}$ at positions $(H, K, L)=\left(q_{x} a / 2 \pi, q_{y} b / 2 \pi, q_{z} c / 2 \pi\right)$ in reciprocal lattice units (rlu), where $a=4.83 \AA . \AA, b=5.75 \AA$, $c=4.99 \AA$. We aligned the crystal in several scattering planes such that the spin-wave (SW) dispersion along the $[1,0,-2],[1,0,2]$ and $[0,1,0]$ symmetric directions that pass across the magnetic Bragg peaks can be readily measured. The incident neutrons with wavelength of $\lambda=4.4 \AA$ were chosen to ensure the needed energy resolution to separate various magnetic excitation branches.

$\mathrm{MnWO}_{4}$ orders in the collinear phase with two inequivalent commensurate wavevectors $q_{M}=(1 / 4,1 / 2, \pm 1 / 2)$. Figures 2(a) and 2(b) present the spin excitation spectra along the $[1,0,2]$ direction with $K=0.5$ and 1.0. This scanning direction goes through the magnetic Bragg peak $(1 / 4,1 / 2,1 / 2)$. The data clearly show four distinct branches that disperse out from the magnetic zone center $(\mathrm{ZC})$ to the zone boundary. The spectra reveal a spin gap of $0.5 \mathrm{meV}$ and boundary energy around $2.2 \mathrm{meV}$. The excitation bandwidth is consistent with the energy
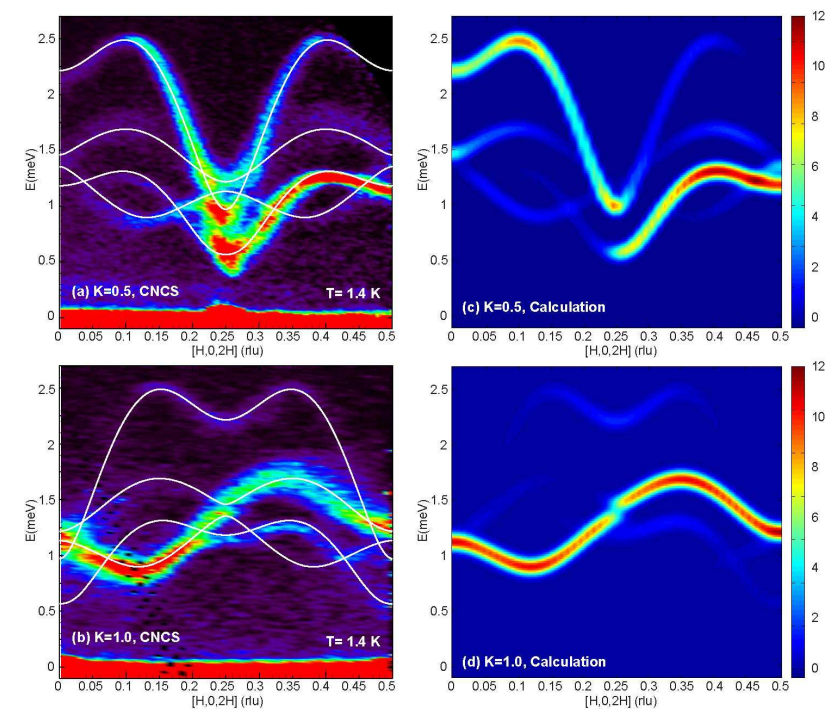

FIG. 2: (Color online) (a) SW dispersion spectra along the $[1,0,2]$ direction through the magnetic peak $(1 / 4,1 / 2,1 / 2)$. (b) Magnetic excitation spectra along the same direction with $K=1.0$. (c and d) Calculated spectra along [1,0,2] direction with $K=0.5$ and $K=1.0$ using magnetic exchange interactions up to $J_{11}$ with instrument resolution convoluted. The solid lines overlapped with experimental data in (a) and (b) are the predicted dispersion curves.

scale of the ordering temperature of $13.5 \mathrm{~K}$. The intensity of the excitation spectra is highly asymmetric with respect to the magnetic Bragg point. For example, the spectral weight of lowest branch in Fig. 2(a) is completely missing as $H$ approaches zero, while it shows the highest intensity as it moves toward $H=0.5$. This highlights the importance of a complete survey in reciprocal space to fully map out the magnetic dynamics.

Figures 3(a) and 3(b) display the magnetic scattering spectra along the $[1,0,-2]$ direction that crosses the other magnetic wavevector $(1 / 4,1 / 2,-1 / 2)$, while Figs. 4(a) and $4(\mathrm{~b})$ illustrate the spectra along the $[0,1,0]$ direction. The icattering intensity map shows similar asymmetric feature on both sides of the magnetic ZC. Investigations along those two directions have been previously reported, $\stackrel{27}{r}$ but the lack of spectral weight for certain branches makes a correct description of the SW dispersion difficult.

The SW dispersion curves can be modeled by a general effective Heisenberg Hamiltonian:

$$
H=-\sum_{i, j} J_{i, j} \mathbf{S}_{i} \cdot \mathbf{S}_{j}-D \sum_{i} S_{i z}^{2},
$$

where $\sum_{i, j}$ indicates summation over pairs of spins,, 30 $D$ is the single-ion anisotropy, and $S_{i z}$ denote the spin components along the easy axis. To calculate the corresponding spectral weight, the low- $T$ spin structure has been verified by collecting a complete set of magnetic reflections that covers the entire reciprocal space. The spin configuration obtained by Rietveld refinement with 
TABLE I: Magnetic exchange coupling parameters derived from spin wave model calculation according to the Eq. $1 . \mathrm{The}^{2+}$ ions located at position $(1 / 2,0.685,1 / 4)$ interact with neighboring spins through one or two oxygens. The bonding distance (in units of $\AA$ ) between Mn...Mn are also listed. The magnetic interaction constants from previous experimental and theoretical studies have been normalized in units of meV for comparison.

\begin{tabular}{|c|c|c|c|c|c|c|c|c|c|c|c|c|c|}
\hline & $J_{1}$ & $J_{2}$ & $J_{3}$ & $J_{4}$ & $J_{5}$ & $J_{6}$ & $J_{7}$ & $J_{8}$ & $J_{9}$ & $J_{10}$ & $J_{11}$ & $D$ & $\chi^{2}$ \\
\hline Mn. & 3.286 & 4.398 & 4.830 & 4.990 & 5.760 & 5.801 & 5.883 & 6.496 & 6.569 & 6.875 & 7.013 & & \\
\hline This work & $-0.47(1)$ & $-0.05(1)$ & $-0.48(1)$ & $-0.21(1)$ & $0.09(1)$ & $-0.49(1)$ & $-0.12(1)$ & $0.05(1)$ & $-0.23(1)$ & -- & -- & $0.12(1)$ & 2.62 \\
\hline This work & $-0.42(1)$ & $-0.04(1)$ & $-0.32(1)$ & $-0.26(1)$ & $0.05(1)$ & $-0.43(1)$ & $-0.12(1)$ & $0.02(1)$ & $-0.26(1)$ & $-0.15(1)$ & $0.02(1)$ & $0.09(1)$ & 1.11 \\
\hline Ref. 27 & -0.084 & -0.058 & -0.182 & 0.178 & 0.009 & -0.219 & 0.010 & 0.212 & -0.980 & -- & -- & 0.061 & -- \\
\hline Ref. 28 & -0.160 & -0.016 & -0.153 & -0.232 & -0.018 & -0.089 & -0.185 & -0.031 & -0.115 & -- & -- & -- & -- \\
\hline
\end{tabular}
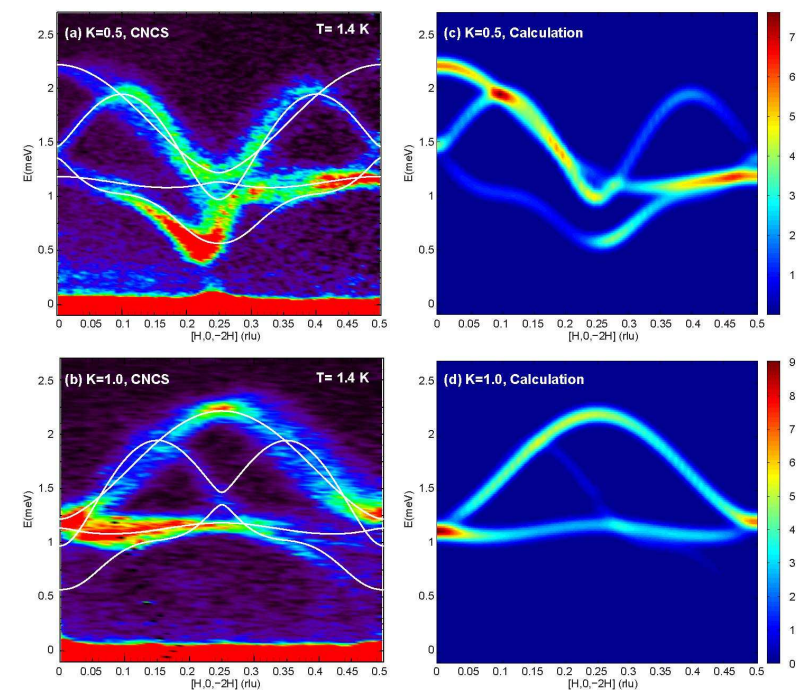

FIG. 3: (Color online) (a) SW dispersion spectra along the $[1,0,-2]$ direction through the magnetic peak $(1 / 4,1 / 2,-1 / 2)$. (b) Magnetic excitation spectra along the same direction with $K=1.0$. (c)-(d) Calculated spectra along the same symmetric directions with $K=0.5$ and $K=1.0$.

group theory analysis is in good agreement with a previous report 23 This configuration is then used to evaluate the magnetic scattering cross-section:

$$
\frac{d^{2} \sigma}{d \Omega d E} \propto f^{2}(Q) e^{-2 W} \sum_{\alpha \beta}\left(\delta_{\alpha \beta}-\hat{Q}_{\alpha} \hat{Q}_{\beta}\right) S^{\alpha \beta}(\mathbf{Q}, \omega)
$$

where $f^{2}(Q)$ is the magnetic form factor for the $\mathrm{Mn}^{2+}$ ion, $e^{-2 W}$ is the Debye-Waller factor, $\hat{Q}_{\alpha}$ is the $\alpha$ component of a unit vector in the direction of $\mathbf{Q}$, and $S^{\alpha \beta}(\mathbf{Q}, \omega)$ is the response function that describes the $\alpha \beta$ spin-spin correlations.

Combining data along all symmetry directions, the dispersion relations can be simultaneously modeled using Eq. (1). We started with nine exchange parameters and single-ion anisotropy $D$, as assumed in Ref. 27. Although the fitting parameters provide a fair description of the data along the $[1,0,-2]$ and $[0,1,0]$ direction, they fail to capture the dispersion relations along the $[1,0,2]$ direc-
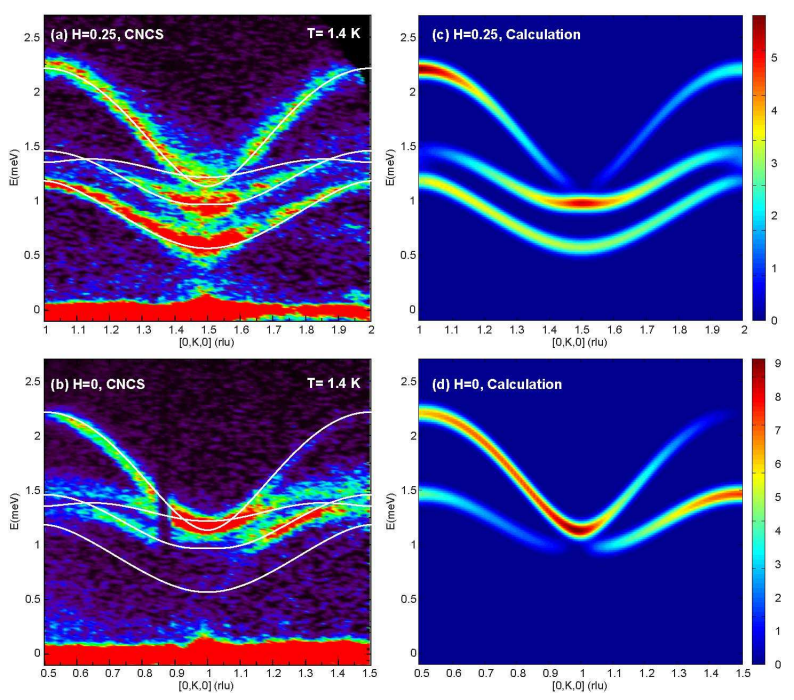

FIG. 4: (Color online) (a) SW dispersion spectra along the $[0,1,0]$ direction through the magnetic peak $(1 / 4,1 / 2,-1 / 2)$. (b) Magnetic excitation spectra along the same direction through the nuclear peak $(0,1,0)$. (c)-(d) Calculated spectra along the same symmetric directions with $H=0.25$ and $H=0$.

tion. Instead, an extra pair of magnetic interactions $J_{10}$ and $J_{11}$ have to be included to achieve satisfactory agreement for the data along all scan directions. As listed in Table I, the magnetic exchange constants generally decrease in amplitude as the bonding distances increase, except the weaker $J_{2}$ and $J_{5}$ (both close to zero) along the $b$ axis. $\mathrm{Mn}^{2+}$ has an electronic configuration of $3 d^{5}$ (orbital singlet) that is not expected to have any magnetic anisotropy, and the nonvanishing $D=0.09 \mathrm{meV}$ indicates a possible spin-orbit coupling that causes the pinning of the magnetic moments in the $a c$ plane ${ }^{29}$ To test whether those parameters are consistent with the actual spin structure, the magnetic energy for all possible spin configurations $\left(2^{8}=256\right.$ with eight spins in one magnetic unit cell) are calculated. We verified that only the spin structure depicted in Fig. 1(a) gives the lowest energy. In addition, we employed the spin-rotation technique to calculate the expected intensity for the SW modes 31 and to compare with the observed wavevector dependence of 
the spectra weight. The right panels of Figs. 2-4 show the excitation spectra map using Eq. (2) with fitted parameters. The excellent agreement between the calculated intensities and the experimental data provides convincing evidence that the formation of the collinear spin structure indeed requires longer range magnetic interactions.

A complex spin configuration would form in the conventional $1 \mathrm{D}$ frustrated spin chain if the next-nearestneighbor antiferromagnetic (AFM) interaction $J^{\prime}$ becomes substantially stronger compared to the nearestneighbor (NN) ferromagnetic (FM) exchange coupling $J$. A spiral phase might appear for $J^{\prime} /|J|>1 / 4$ and a collinear $\uparrow \uparrow \downarrow \downarrow$ structure will emerge when $J^{\prime} /|J|>1 / 2 . \underline{3}$ The zigzag $E$-type phase observed in $R \mathrm{MnO}_{3}$ is a classic example of competing short-range FM-AFM interactions caused by lattice distortions. ${ }^{32,33}$ In the case the $\mathrm{MnWO}_{4}$, the exchange coupling are predominantly AFM and three-dimensional. Nevertheless, strong longrange magnetic interactions comparable to $\mathrm{NN}$ interaction $\left(J_{1}\right)$ along the $c$ axis $\left(\left|J_{4} / J_{1}\right|>1 / 2\right)$ and $a$ axis $\left(\left|J_{3} / J_{1}\right|>1 / 2,\left|J_{6} / J_{1}\right| \approx 1\right.$ and $\left.\left|J_{7} / J_{1}\right|>1 / 4\right)$ are observed. This reflects the magnetic frustration in those two directions and is consistent with the ICM components present in the $a$ and $c$ directions but not in the $b$ direction, when the system enters the spiral phase. The exchange coupling remains sizable $\left(J_{10}\right)$ even at a rather long distance. Such an unusual extended interaction could also be viewed as a much-reduced NN exchange coupling because of a nearly $90^{\circ} \mathrm{Mn}-\mathrm{O}-\mathrm{Mn}$ bonding angle. 34 Thus, the unique crystalline structure makes $\mathrm{MnWO}_{4}$ a promising material to achieve novel physical properties when the magnetic interactions are modified. It was reported that doping a few percent magnetic or nonmagnetic impurities can drastically affect the spin order ${ }^{17-20}$ For instance, while replacing $\mathrm{Mn}^{2+}$ with $\mathrm{Fe}^{2+}$ ions that have a larger local magnetic anisotropy seems to enhance the collinear structure $\stackrel{17}{\underline{17}}$ the introduction of nonmagnetic $\mathrm{Zn}^{2+}$ ions that weaken the overall magnetic interactions switches the ground state from collinear to spiral order $\frac{18,20}{}$ Those results demonstrate that chemical substitutions are a viable tool to tune the multiferroic properties in the extremely sensitive $\mathrm{MnWO}_{4}$.

In summary, high-resolution INS is used to study the SWs in the collinear phase of $\mathrm{MnWO}_{4}$. The collinear spin order is stabilized by the delicate balance of competing long-range magnetic interactions. We provide an effective Hamiltonian to describe the highly frustrated magnetic order within the zigzag spin chains along the $c$ axis and between spin chains along $a$ axis. Rich and complex magnetic phases are expected in chemically doped $\mathrm{MnWO}_{4}$ due to the fine-tuning of the magnetic interactions. The delicate balance of exchange interactions in $\mathrm{MnWO}_{4}$ can also be used to achieve magnetoelectric control using external electric or magnetic fields.

This work was partially supported by the Division of Scientific User Facilities of the Office of Basic Energy Sciences, U.S. Department of Energy. Work at Houston is supported in part by the T.L.L. Temple Foundation, the J. J. and R. Moores Endowment, and the state of Texas through TCSUH and at LBNL through the US DOE, Contract No. DE-AC03-76SF00098.
* Electronic address: yef1@ornl.gov

1 Y. Tokura, Science 312, 1481 (2006).

2 D. I. Khomskii, J. Magn. Magn. Mater. 306, 1 (2006).

3 S.-W. Cheong and M. Mostovoy, Nature Materials 6, 13 (2007).

4 T. Kimura, Annu. Rev. Mater. Res. 37, 387 (2007).

5 T. Kimura, J. C. Lashley, and A. P. Ramirez, Phys. Rev. B 73, 220401(R) (2006).

${ }^{6}$ K. Kimura, H. Nakamura, K. Ohgushi, and T. Kimura, Phys. Rev. B 78, 140401(R) (2008).

7 M. Kenzelmann et al., Phys. Rev. Lett. 98, 267205 (2007).

8 G. Lawes et al., Phys. Rev. Lett. 95, 087205 (2005).

${ }^{9}$ Kimura et al., Nature (London) 426, 55 (2005).

10 T. Goto, T. Kimura, G. Lawes, A. P. Ramirez, and Y. Tokura, Phys. Rev. Lett. 92, 257201 (2004).

11 N. Hur et al., Nature (Lodon) 429, 392 (2004).

12 Y. Yamasaki et al., Phys. Rev. Lett. 98, 147204 (2007).

13 K. Taniguchi, N. Abe, T. Takenobu, Y. Iwasa, and T. Arima, Phys. Rev. Lett. 97, 097203 (2006).

14 O. Heyer et al., J. Phys.: Condens. Matter 18, L471 (2006).

15 A. H. Arkenbout, T. T. M. Palstra, T. Siegrist, and T. Kimura, Phys. Rev. B 74, 184431 (2006).

16 H. Sagayama et al., Phys. Rev. B 77, 220407(R) (2008).

17 F. Ye et al., Phys. Rev. B 78, 193101 (2008).

18 L. Meddar et al., Chem. Mater. 21, 5203 (2009).
19 Y.-S. Song, J.-H. Chung, J. M. S. Park, and Y.-N. Choi, Phys. Rev. B 79, 224415 (2009).

20 R. P. Chaudhury et al., Phys. Rev. B 83, 014401 (2011).

21 T. Finger et al., Phys. Rev. B 81, 054430 (2010).

${ }^{22}$ K. Taniguchi, N. Abe, H. Umetsu, H. A. Katori, and T. Arima, Phys. Rev. Lett. 101, 207205 (2008).

${ }^{23}$ G. Lautenschläger et al., Phys. Rev. B 48, 6087 (1993).

24 H. Katsura, N. Nagaosa, and A. V. Balatsky, Phys. Rev. Lett. 95, 057205 (2005).

25 I. A. Sergienko and E. Dagotto, Phys. Rev. B 73, 094434 (2006).

26 M. Mostovoy, Phys. Rev. Lett. 96, 067601 (2006).

27 H. Ehrenberg, H. Weitzel, H. Fuess, and B. Hennion, J. Phys.: Condens. Matter 11, 2649 (1999).

28 C. Tian et al., Phys. Rev. B 80, 104426 (2009).

29 N. Hollmann et al., Phys. Rev. B 82, 184429 (2010).

30 Spin pairs up to 11 th $\mathrm{NN}$ are included to calcuate the dispersion relation. This limits magnetic coupling between $\mathrm{Mn}$ ions through zero or one $\mathrm{WO}_{6}$ octahedron.

31 J. T. Haraldsen and R. S. Fishman, J. Phys.: Condens. Matter 21, 216001 (2009).

32 T. Kimura et al., Phys. Rev. B 68, 060403(R) (2003).

33 M. Mochizuki, N. Furukawa, and N. Nagaosa, Phys. Rev. Lett. 105, 037205 (2010).

34 J. B. Goodenough, Magnetism and Chemical Bond, (Interscience, New York, 1963). 